\title{
Propargylation of Aldehydes Using Potassium Allenyltrifluoroborate
}

\author{
Túlio R. Couto ${ }^{a}$ \\ Jucleiton J. R. Freitas ${ }^{\mathrm{a}}$ \\ Juliano C. R. Freitas ${ }^{b}$ \\ Italo H. Cavalcantia \\ Paulo H. Menezes ${ }^{a}$ \\ Roberta A. Oliveira*a \\ a Departamento de Química Fundamental, Universidade Federal \\ de Pernambuco, 50740-540 Recife-PE, Brazil \\ roberta.ayres@pq.cnpq.br \\ ' Unidade de Educação e Saúde, Universidade Federal de Campina \\ Grande, 58175-000 Cuité-PB, Brazil
}

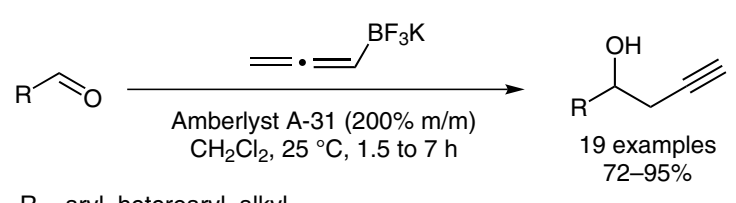

$\mathrm{R}=$ aryl, heteroaryl, alkyl
Received: 28.05.2014

Accepted after revision: 26.08.2014

Published online: 25.09 .2014

DOI: 10.1055/s-0034-1379163; Art ID: ss-2014-m0329-op

Abstract The commercially available resin Amberlyst A-31 was efficiently used to promote the propargylation of aldehydes using potassium allenyltrifluoroborate. The method is simple and fast, and the products were obtained in short reaction times in high yields and purity at room temperature in a regio- and chemoselective manner.

Key words propargylation, potassium organotrifluoroborates, Amberlyst A-31

The propargylation of carbonyl compounds is an important reaction in organic synthesis. ${ }^{1}$ Usually, it involves the use of an appropriate propargyl or allenyl organometallic compound or the direct propargylic substitution of propargyl alcohols or their derivatives with nucleophiles. ${ }^{2}$

The reactions involving the direct addition of an allenyl or propargyl organometallic reagent to a carbonyl compound usually proceed through an $\mathrm{S}_{\mathrm{E}} 2$-type mechanism. ${ }^{3}$ On the other hand, some reactions are based on the in situ formation of the propargyl or allenyl organometallic species, which can interconvert, followed by the subsequent addition to the appropriate carbonyl compound. In this case, the regioselectivity of the reaction is usually governed by the rate of isomerization, stability, and the nucleophilicity of propargyl or allenyl species involved in the reaction. ${ }^{3}$

The development of propargyl nucleophiles that are able to form new $\mathrm{C}-\mathrm{C}$ bonds under mild conditions in a very regioselective manner is a subject of great interest and a variety of propargyl or allenyl organometallics derived from zinc, ${ }^{4}$ titanium, ${ }^{5}$ aluminum, ${ }^{6}$ lithium, ${ }^{7}$ and magnesium, ${ }^{8}$ were developed for this purpose. In addition, different silicon, ${ }^{9}$ tin, ${ }^{10}$ and boron derivatives ${ }^{11}$ were successfully employed in this reaction.
The use of less reactive compounds such as allenylsilanes and allenylstannanes requires Lewis acid as additives. Although the utility of allenylstannanes is further indicated by the commercial availability of some of them, the toxicity of these compounds makes them inappropriate for use in pharmaceutical synthesis. ${ }^{12}$ Moreover, the removal of tributyltin residues from reaction mixtures is also a major issue.

The use of organoboranes is limited to their compatibility to functional groups and sensitivity to air and moisture. Conversely, boronic acids are known for their difficulty to purify and the uncertainty in the stoichiometry. ${ }^{13}$ This problem can be circumvented by converting these into their corresponding boronate esters, ${ }^{14}$ which is a more stable alternative, but lacks atom economy. In addition, this class of compounds has low hydrolytic stability, which is dependent on the kind of alcohol used for its preparation. ${ }^{15}$

The use of potassium organotrifluoroborates seems to be the best option due to their stability, which also allows the complete characterization of these salts by heteronuclei NMR analysis, ${ }^{16}$ and exact mass measurements. ${ }^{17}$ Additionally, a marked increase in atom economy, ${ }^{18}$ stability, and the apparent low toxicity ${ }^{19}$ of organotrifluoroborate salts make them more appealing.

Recently, we have described the use of the commercially available resin Amberlyst-15 as an efficient promoter for the allylation of aldehydes using potassium allyltrifluoroborate. ${ }^{20}$ Herein, we describe the synthesis of homopropargylic alcohols from the reaction of potassium allenyltrifluoroborate and aldehydes containing different functional groups. To our knowledge, this is the first method for propargylation of aldehydes based on the use of potassium organotrifluoroborates.

In the course of developing an optimal set of reaction conditions, the amount of resin and the type of solvent were first examined to promote the reaction. Thus, 4-nitrobenzaldehyde (1a; $1 \mathrm{mmol}$ ) and potassium allenyltrifluo- 
roborate $(2 ; 1.7 \mathrm{mmol})$ were treated at room temperature using Amberlyst A-15 and the progress of the reaction was monitored by TLC. The results are presented in Table 1.

Table 1 Effect of Amberlyst A-15 on the Allylation of 4-Nitrobenzaldehyde by Potassium Allenyltrifluoroborate ${ }^{\text {a }}$

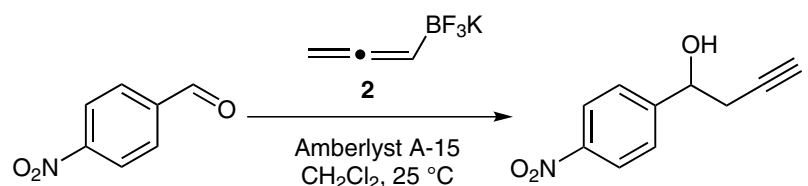

1a

$3 a$

\begin{tabular}{lllll}
\hline Entry & Amberlyst A-15 $(\% \mathrm{~m} / \mathrm{m})$ & Solvent & Time $(\mathrm{h})$ & $\mathbf{3 a}(\%)^{\mathrm{b}}$ \\
\hline 1 & 100 & $\mathrm{EtOH}$ & 16.5 & 33 \\
2 & 100 & $\mathrm{H}_{2} \mathrm{O}$ & 16.5 & 6 \\
3 & 100 & $\mathrm{CH}_{2} \mathrm{Cl}_{2}$ & 16.5 & 21 \\
4 & 200 & $\mathrm{EtOH}$ & 5.0 & 20 \\
5 & 200 & $\mathrm{H}_{2} \mathrm{O}$ & 5.0 & 5 \\
6 & 200 & $\mathrm{CH}_{2} \mathrm{Cl}_{2}$ & 3.0 & 90 \\
\hline
\end{tabular}

a Reaction conditions: reactions were performed with $\mathbf{1 a}(1 \mathrm{mmol})$ and $\mathbf{2}$

$(1.7 \mathrm{mmol})$ in solvent $(5 \mathrm{~mL})$ at $25^{\circ} \mathrm{C}$ for the time indicated.

$\mathrm{b}$ The conversion was determined by GC with respect to $1 \mathbf{a}$.

When a $100 \% \mathrm{~m} / \mathrm{m}$ amount of Amberlyst A-15 was used, the corresponding low conversions were observed. When water was used as the reaction solvent, the corresponding product 3a was obtained in low yield after 16.5 hours (Table 1 , entry 2 ). This result can probably be explained by the low solubility of aldehyde 1a in water. A similar behavior was observed when dichloromethane was used as the reaction solvent (Table 1, entry 3) where the formation of $\mathbf{3 a}$ was observed to only $21 \%$, probably due to the low solubility of potassium allenyltrifluoroborate $\mathbf{2}$ in dichloromethane. When ethanol was used as the reaction solvent, lower conversion to 3a was observed together with the acid-catalyzed ketalization product of the aldehyde (Table 1 , entry 1 ).

A dramatic effect was observed when the amount of the promoter was increased to $200 \% \mathrm{~m} / \mathrm{m}$. In this case, a higher conversion of the aldehyde $\mathbf{1 a}$ into the product $\mathbf{3 a}$ was observed when dichloromethane was used as the reaction solvent (Table 1 , entry 6 ).

Next, we examined a variety of commercially available resins to promote the propargylation of 4-nitrobenzaldehyde (1a; 1.0 equiv) by potassium allenyltrifluoroborate ( $\mathbf{2}$; 1.7 equiv) using dichloromethane as the reaction solvent at room temperature (Table 2 ).

In the absence of a promoter, the corresponding product 3a was obtained in low yield after 24 hours (Table 2, entry 1). When acidic resins were used, higher conversions of 1a into the corresponding product 3a were observed (Table 2 , entries 2, 3, 6-10). The best result was obtained when Amberlyst A-31 was used as the promoter (Table 2 , entry 6 ). In-
Table 2 Comparative Efficiency of Various Resins in the Addition of Potassium Allenyltrifluoroborate to 4-Nitrobenzaldehyde ${ }^{\text {a }}$

\begin{tabular}{llll} 
Resin $(200 \% \mathrm{~m} / \mathrm{m})$ & Time (h) & 3a $)^{\mathrm{b}}$ \\
\hline 1 & none & 24.0 & 19 \\
2 & Amberlyst A-15 & 3.0 & 90 \\
3 & Amberlyst A-16 & 3.0 & 99 \\
4 & Amberlyst A-21 & 3.0 & - \\
5 & Amberlyst A-26 & 3.0 & - \\
6 & Amberlyst A-31 & 1.5 & 99 \\
7 & Amberlyst A-35 & 3.0 & 99 \\
8 & Amberlyst A-36 & 3.0 & 76 \\
9 & Amberlyst A-40 & 3.0 & 93 \\
10 & Amberlyst A-41 & 3.0 & 85 \\
\hline
\end{tabular}

a Reaction conditions: reactions were performed with $\mathbf{1 a}(1 \mathrm{mmol})$ and $\mathbf{2}$ (1.7 mmol) using the appropriate resin in $\mathrm{CH}_{2} \mathrm{Cl}_{2}(5 \mathrm{~mL})$ at $25^{\circ} \mathrm{C}$ for the time indicated.

b The conversion was determined by GC with respect to $\mathbf{1 a}$.

terestingly, when the basic resins Amberlyst A-21 and A-26, were used, the corresponding propargylation product $\mathbf{3 a}$ was not observed in either case (Table 2, entries 4 and 5).

The effect of the amount of resin to promote the reaction was also investigated. The load of Amberlyst A-31 was varied from 50 to $400 \% \mathrm{~m} / \mathrm{m}$ (Table 3 ). It was observed that the reaction yield changed appreciably when the amount of the resin varied from 50 to $200 \% \mathrm{~m} / \mathrm{m}$ (Table 3 , entries $1-3$ ) after 1.5 hours. However, higher amounts did not change the reaction yield considerably (Table 3 , entry 4 ).

Table 3 Allylation of 4-Nitrobenzaldehyde by Potassium Allenyltrifluoroborate Using Different Amounts of Amberlyst A-31 ${ }^{\text {a }}$

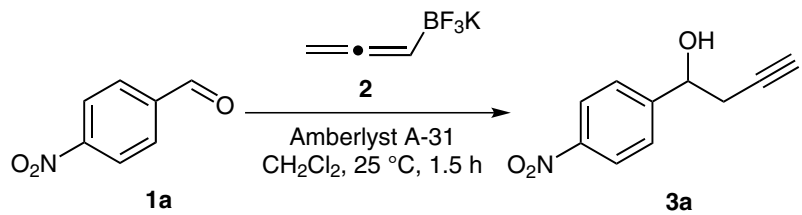

\begin{tabular}{|c|c|c|}
\hline Entry & Amberlyst A-31 (\% m/m) & $3 a(\%)^{b}$ \\
\hline 1 & 50 & 0 \\
\hline 2 & 100 & 7 \\
\hline 3 & 200 & 99 \\
\hline 4 & 400 & 91 \\
\hline
\end{tabular}


The optimized reaction conditions, namely: potassium allenyltrifluoroborate $\mathbf{2}(1.7 \mathrm{mmol})$, aldehyde $(1 \mathrm{mmol})$ and Amberlyst A-31 $(200 \% \mathrm{~m} / \mathrm{m})$ in dichloromethane $(5 \mathrm{~mL})$, were then applied in the propargylation reaction of alde- hydes containing a wide range of functional groups. Thus, aliphatic, aromatic, $\alpha, \beta$-unsaturated, and heterocyclic aldehydes were efficiently propargylated in high yields (Table 4).

Table 4 Propargylation of Aldehydes with Potassium Allenyltrifluoroborate ${ }^{\mathrm{a}}$

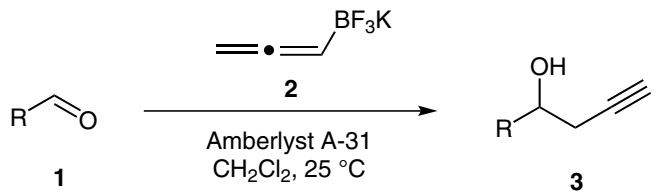

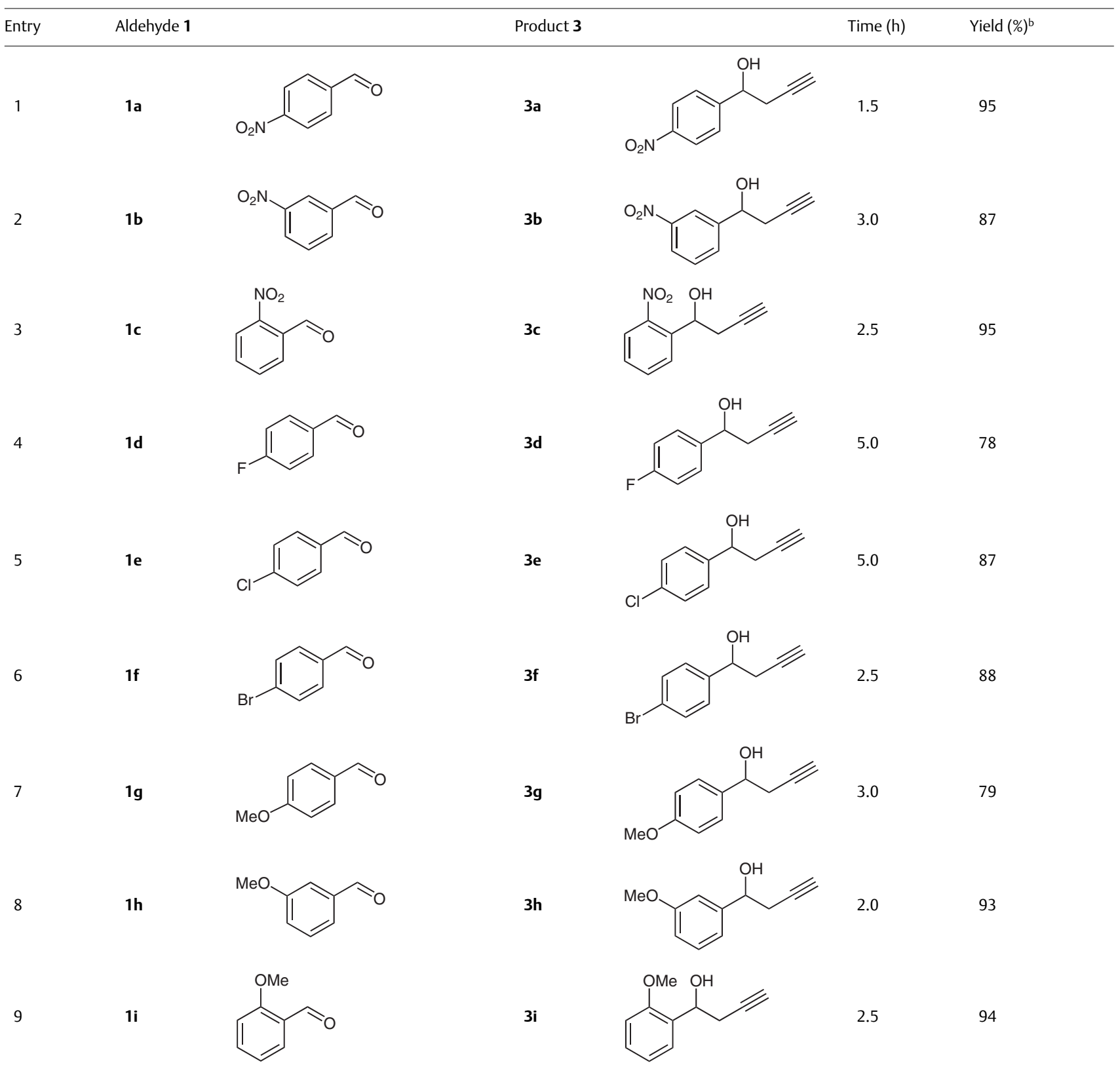


Synthesis

T. R. Couto et al.

Paper

Table 4 (continued)

\begin{tabular}{|c|c|c|c|c|}
\hline Entry & Aldehyde 1 & Product 3 & Time (h) & Yield (\%) ${ }^{\mathrm{b}}$ \\
\hline 10 & $1 \mathrm{j}$ & $3 \mathbf{j}$ & 7.0 & 72 \\
\hline 11 & $1 \mathrm{k}$ & $3 k$ & 2.5 & 95 \\
\hline 12 & 11 & 31 & 3.0 & 73 \\
\hline 13 & $1 \mathrm{~m}$ & $3 m$ & 7.0 & 90 \\
\hline 14 & $1 n$ & $3 n$ & 3.0 & 80 \\
\hline 15 & 10 & 30 & 2.5 & 82 \\
\hline 16 & $1 p$ & $3 p$ & 2.0 & 85 \\
\hline 17 & $1 q$ & $3 q$ & 5.0 & 70 \\
\hline 18 & $1 r$ & $3 r$ & 2.0 & 92 \\
\hline 19 & $1 \mathrm{~s}$ & $3 s$ & 2.0 & 94 \\
\hline
\end{tabular}

a Reaction conditions: reactions were performed with the appropriate aldehyde $\mathbf{1}(1 \mathrm{mmol})$ and $\mathbf{2}(1.7 \mathrm{mmol})$ using Amberlyst A-31 $(200 \% \mathrm{~m} / \mathrm{m})$ in $\mathrm{CH}{ }_{2} \mathrm{Cl}_{2}(5 \mathrm{~mL})$ at $25^{\circ} \mathrm{C}$ for the time indicated.

${ }^{\mathrm{b}}$ Isolated yields. 
The effect of substituents on the aromatic ring has little influence in the yield. The propargylation of aldehydes containing electron-withdrawing groups gave the corresponding products in high yields (Table 4, entries 1-6). Electronrich aldehydes, $\beta$-naphthaldehyde, benzaldehyde, and a heterocyclic aldehyde led to the homopropargylic alcohols also in high yields (Table 4, entries 7-12).

The reaction is regioselective while only the 1,2 -addition product was observed when an $\alpha, \beta$-unsaturated aldehyde was used (Table 4, entry 13). For aliphatic aldehydes, the propargylation method also exhibited high efficiency (Table 4, entry 14). The chemoselectivity of the method was evaluated using different functionalized aldehydes. In all cases, the corresponding products were selectively obtained in good yields (Table 4, entries 15-19).

The recoverability and recyclability of the resin were also investigated. Thus, after each run, the catalyst was separated from the reaction mixture, washed with dichloromethane, and reused. It was found that the resin could be recovered and reused in further propargylation reactions, however, the conversion of 4-nitrobenzaldehyde 1a into the propargylation product 3a significantly decreased after the third run (Table 5).

Table 5 Amberlyst A-31 $\left(200 \%\right.$ m/m) Recycling after Successive Runs ${ }^{\mathrm{a}}$

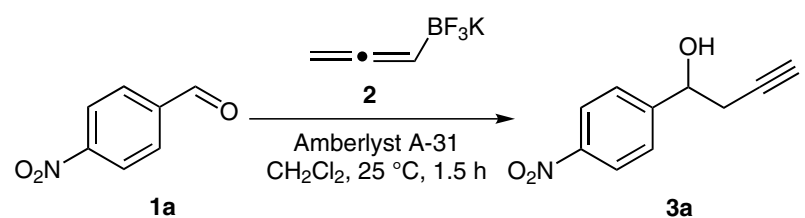

\begin{tabular}{|c|c|c|}
\hline Run & Time (h) & $3 a(\%)^{b}$ \\
\hline 1 & 1.5 & 99 \\
\hline 2 & 3.0 & 87 \\
\hline 3 & 3.0 & 70 \\
\hline 4 & 3.0 & 9 \\
\hline 5 & 3.0 & 0 \\
\hline \multicolumn{3}{|c|}{$\begin{array}{l}\text { a Reaction conditions: reactions were performed with } \mathbf{1 a}(1 \mathrm{mmol}) \text { and } \mathbf{2} \\
(1.7 \mathrm{mmol}) \text { using Amberlyst } \mathrm{A}-31(200 \% \mathrm{~m} / \mathrm{m}) \text { in } \mathrm{CH}_{2} \mathrm{Cl}_{2}(5 \mathrm{~mL}) \text { at } 25^{\circ} \mathrm{C} \text { for } \\
\text { the time indicated. } \\
\text { b The conversion was determined by } \mathrm{GC} \text { with respect to } \mathbf{1 a} \text {. }\end{array}$} \\
\hline
\end{tabular}

In summary, we have shown that the resin Amberlyst A31 is an efficient promoter for the propargylation of aldehydes using potassium allenyltrifluoroborate. The method features the use of a commercially available resin, and the products were obtained in short reaction times in high yield and purity at room temperature. The method is simple, fast and efficient and could be applied for the synthesis of more complex compounds.
${ }^{1} \mathrm{H}$ NMR and ${ }^{13} \mathrm{C}$ NMR data were recorded in $\mathrm{CDCl}_{3}$ or DMSO- $d_{6}$. The chemical shifts are reported as delta $(\delta)$ units in parts per million (ppm) relative to the solvent residual peak as the internal reference. ${ }^{11} \mathrm{~B}(128 \mathrm{MHz})$ NMR spectra were recorded in $\mathrm{D}_{2} \mathrm{O}$ and ${ }^{19} \mathrm{~F}(376 \mathrm{MHz})$ in DMSO- $d_{6}$. Spectra were calibrated using $\mathrm{Et}_{2} \mathrm{O} \cdot \mathrm{BF}_{3}(0.0 \mathrm{ppm})$ as external reference in the case of ${ }^{11} \mathrm{~B}$ NMR and chemical shifts were referenced to external $\mathrm{CF}_{3} \mathrm{CO}_{2} \mathrm{H}(0.0 \mathrm{ppm})$ in the case of ${ }^{19} \mathrm{~F}$ NMR spectra. Coupling constants $(J)$ for all spectra are reported in hertz $(\mathrm{Hz})$. Reactions were monitored by TLC on $0.25 \mathrm{~mm}$ E. Merck silica gel 60 plates (F254) using UV light, vanillin, and p-anisaldehyde as visualizing agents.

The preparation of potassium allenyltrifluoroborate $(\mathbf{2})$ is described in the Supporting Information.

Propargylation of Aldehydes 1 with Potassium Allenyltrifluoroborate (2) Using Amberlyst A-31; General Procedure

To a solution of the appropriate aldehyde $\mathbf{1}(1.0 \mathrm{mmol})$ in $\mathrm{CH}_{2} \mathrm{Cl}_{2}(5$ $\mathrm{mL}$ ) was added Amberlyst A-31 $(200 \% \mathrm{~m} / \mathrm{m})$ followed by potassium allenyltrifluoroborate $(2 ; 248 \mathrm{mg}, 1.70 \mathrm{mmol})$. The mixture was stirred for the time indicated in Table 4 and then diluted with $\mathrm{CH}_{2} \mathrm{Cl}_{2}$ ( $5 \mathrm{~mL})$ and washed with $\mathrm{H}_{2} \mathrm{O}(2 \times 15 \mathrm{~mL})$. The aqueous layer was extracted with $\mathrm{CH}_{2} \mathrm{Cl}_{2}(2 \times 5 \mathrm{~mL})$. The combined organic layers were dried $\left(\mathrm{MgSO}_{4}\right)$, filtered, and the solvent was removed under reduced pressure to yield $\mathbf{3}$ without the need for further purification.

\section{1-(4-Nitrophenyl)but-3-yn-1-ol (3a)}

Yield: $183 \mathrm{mg}(95 \%)$; white solid; $\mathrm{mp} 116-118^{\circ} \mathrm{C}$.

${ }^{1} \mathrm{H} \mathrm{NMR}\left(400 \mathrm{~Hz}, \mathrm{CDCl}_{3}\right): \delta=8.23\left(\mathrm{~d}, J=8.4 \mathrm{~Hz}, 2 \mathrm{H}_{\mathrm{arom}}\right), 7.59(\mathrm{~d}, J=8.4$ $\left.\mathrm{Hz}, 2 \mathrm{H}_{\mathrm{arom}}\right), 5.00$ (dd, $\left.J=7.2,6.0 \mathrm{~Hz}, 1 \mathrm{H}, \mathrm{OCHCH}_{2}\right), 2.72(\mathrm{ddd}, J=16.8$, 6.0, $2.8 \mathrm{~Hz}, 1 \mathrm{H}, \mathrm{OCHCH}_{2}$ ), 2.63 (ddd, $J=16.8,7.2,2.8 \mathrm{~Hz}, 1 \mathrm{H}$, $\left.\mathrm{OCHCH}_{2}\right), 2.11(\mathrm{t}, J=2.8 \mathrm{~Hz}, 1 \mathrm{H}, \mathrm{C} \equiv \mathrm{CH}), 1.88(\mathrm{br} \mathrm{s}, 1 \mathrm{H}, \mathrm{OH})$.

${ }^{13} \mathrm{C}$ NMR $\left(100 \mathrm{~Hz}, \mathrm{CDCl}_{3}\right): \delta=149.4,147.6,126.6,123.7,79.3,72.0$, 71.3, 29.5 .

The spectra were in accordance with the previously reported data. ${ }^{21}$

\section{1-(3-Nitrophenyl)but-3-yn-1-ol (3b)}

Yield: $168 \mathrm{mg}$ (87\%); yellow oil.

${ }^{1} \mathrm{H} \mathrm{NMR}\left(400 \mathrm{~Hz}, \mathrm{CDCl}_{3}\right): \delta=8.22\left(\mathrm{t}, J=2.0,1 \mathrm{H}_{\mathrm{arom}}\right), 8.09$ (ddd, $J=8.0$, $\left.2.0,0.8 \mathrm{~Hz}, 1 \mathrm{H}_{\text {arom }}\right), 7.69-7.67\left(\mathrm{~m}, 1 \mathrm{H}_{\text {arom }}\right), 7.48\left(\mathrm{t}, J=7.6 \mathrm{~Hz}, 1 \mathrm{H}_{\text {arom }}\right)$, $4.93\left(\mathrm{dd}, J=7.2,5.6 \mathrm{~Hz}, 1 \mathrm{H}, \mathrm{OCHCH}_{2}\right), 2.64-2.60\left(\mathrm{~m}, 2 \mathrm{H}, \mathrm{OCHCH}_{2}\right)$, 2.08 (br s, $1 \mathrm{H}, \mathrm{OH}), 2.04(\mathrm{t}, J=2.8 \mathrm{~Hz}, 1 \mathrm{H}, \mathrm{C} \equiv \mathrm{CH}$ ).

${ }^{13} \mathrm{C}$ NMR $\left(100 \mathrm{~Hz}, \mathrm{CDCl}_{3}\right): \delta=148.3,144.4,131.9,129.4,122.9,120.9$, 79.4, 72.0, 71.2, 29.5.

The spectra were in accordance with the previously reported data. ${ }^{21}$

\section{1-(2-Nitrophenyl)but-3-yn-1-ol (3c)}

Yield: $183 \mathrm{mg}$ (95\%); yellow oil.

${ }^{1} \mathrm{H}$ NMR $\left(400 \mathrm{~Hz}, \mathrm{CDCl}_{3}\right): \delta=7.90\left(\mathrm{dd}, J=8.0,1.2 \mathrm{~Hz}, 1 \mathrm{H}_{\text {arom }}\right), 7.82(\mathrm{dd}$, $\left.J=8.0,1.2 \mathrm{~Hz}, 1 \mathrm{H}_{\text {arom }}\right), 7.61\left(\mathrm{t}, J=8.0 \mathrm{~Hz}, 1 \mathrm{H}_{\text {arom }}\right), 7.40(\mathrm{t}, J=8.0 \mathrm{~Hz}, 1$ $\left.\mathrm{H}_{\text {arom }}\right), 5.41$ (dd, $\left.J=7.6,4.8 \mathrm{~Hz}, 1 \mathrm{H}, \mathrm{OCHCH}_{2}\right), 2.86(\mathrm{ddd}, J=16.4,4.8$, $2.8 \mathrm{~Hz}, 1 \mathrm{H}, \mathrm{OCHCH}_{2}$ ), 2.62 (ddd, $J=16.4,7.6,2.8 \mathrm{~Hz}, 1 \mathrm{H}, \mathrm{OCHCH}_{2}$ ), $2.04(\mathrm{t}, J=2.8 \mathrm{~Hz}, 1 \mathrm{H}, \mathrm{C} \equiv \mathrm{CH})$.

${ }^{13} \mathrm{C}$ NMR $\left(100 \mathrm{~Hz}, \mathrm{CDCl}_{3}\right): \delta=147.8,137.7,133.5,128.6,128.2,124.5$, 79.7, 71.8, 67.4, 28.5.

The spectra were in accordance with the previously reported data. ${ }^{22}$ 


\section{1-(4-Fluorophenyl)but-3-yn-1-ol (3d)}

Yield: $129 \mathrm{mg}$ (78\%); yellow oil.

${ }^{1} \mathrm{H}$ NMR (400 Hz, $\mathrm{CDCl}_{3}$ ): $\delta=7.32-7.28\left(\mathrm{~m}, 2 \mathrm{H}_{\mathrm{arom}}\right), 7.00-6.96(\mathrm{~m}, 2$ $\left.\mathrm{H}_{\text {arom }}\right), 4.80\left(\mathrm{t}, J=6.4 \mathrm{~Hz}, 1 \mathrm{H}, \mathrm{OCHCH}_{2}\right), 2.56(\mathrm{dd}, J=6.4,2.8 \mathrm{~Hz}, 2 \mathrm{H}$, $\left.\mathrm{OCHCH}_{2}\right), 2.01(\mathrm{t}, J=2.8 \mathrm{~Hz}, 1 \mathrm{H}, \mathrm{C} \equiv \mathrm{CH})$.

${ }^{13} \mathrm{C}$ NMR $\left(100 \mathrm{~Hz}, \mathrm{CDCl}_{3}\right): \delta=163.6,138.1,127.5,115.4,80.3,71.7$, 71.2, 29.6.

The spectra were in accordance with the previously reported data. ${ }^{21}$

\section{1-(4-Chlorophenyl)but-3-yn-1-ol (3e)}

Yield: $158 \mathrm{mg}$ (87\%); yellow oil.

${ }^{1} \mathrm{H}$ NMR ( $300 \mathrm{~Hz}, \mathrm{CDCl}_{3}$ ): $\delta=7.27$ (br s, $4 \mathrm{H}_{\text {arom }}$ ), 4.79 (dd, $J=7.2,6.0$ $\left.\mathrm{Hz}, 1 \mathrm{H}, \mathrm{OCHCH}_{2}\right), 2.57-2.54\left(\mathrm{~m}, 2 \mathrm{H}, \mathrm{OCHCH}_{2}\right), 2.08($ br s, $1 \mathrm{H}, \mathrm{OH})$, $2.01(\mathrm{t}, J=2.8 \mathrm{~Hz}, 1 \mathrm{H}, \mathrm{C} \equiv \mathrm{CH})$.

${ }^{13} \mathrm{C}$ NMR $\left(75 \mathrm{~Hz}, \mathrm{CDCl}_{3}\right): \delta=140.8,133.7,128.6,127.2,80.1,71.6$, 71.3, 29.5 .

The spectra were in accordance with the previously reported data. ${ }^{21}$

\section{1-(4-Bromophenyl)but-3-yn-1-ol (3f)}

Yield: $200 \mathrm{mg}$ (88\%); yellow oil.

${ }^{1} \mathrm{H}$ NMR $\left(400 \mathrm{MHz}, \mathrm{CDCl}_{3}\right): \delta=7.42\left(\mathrm{~d}, J=8.4 \mathrm{~Hz}, 2 \mathrm{H}_{\mathrm{arom}}\right), 7.21(\mathrm{~d}, J=$ $\left.8.4 \mathrm{~Hz}, 2 \mathrm{H}_{\text {arom }}\right), 4.78$ (dd, $\left.J=6.8,5.6 \mathrm{~Hz}, 1 \mathrm{H}, \mathrm{OCHCH}_{2}\right), 2.56-2.53(\mathrm{~m}, 2$ $\left.\mathrm{H}, \mathrm{OCHCH}_{2}\right), 2.01(\mathrm{t}, J=2.4 \mathrm{~Hz}, 1 \mathrm{H}, \mathrm{C} \equiv \mathrm{CH})$.

${ }^{13} \mathrm{C}$ NMR $\left(100 \mathrm{MHz}, \mathrm{CDCl}_{3}\right): \delta=141.4,131.6,127.5,121.8,80.1,71.6$, 71.4, 29.4.

The spectra were in accordance with the previously reported data. ${ }^{22}$

\section{1-(4-Methoxyphenyl)but-3-yn-1-ol (3g)}

Yield: $140 \mathrm{mg}$ (79\%); yellow oil.

${ }^{1} \mathrm{H}$ NMR $\left(400 \mathrm{~Hz}, \mathrm{CDCl}_{3}\right): \delta=7.25\left(\mathrm{~d}, J=8.4 \mathrm{~Hz}, 2 \mathrm{H}_{\mathrm{arom}}\right), 6.91(\mathrm{~d}, J=8.4$ $\left.\mathrm{Hz}, 2 \mathrm{H}_{\mathrm{arom}}\right), 4.77\left(\mathrm{t}, J=6.4 \mathrm{~Hz}, 1 \mathrm{H}, \mathrm{OCHCH}_{2}\right), 3.74\left(\mathrm{~s}, 3 \mathrm{H}, \mathrm{OCH}_{3}\right), 2.58-$ $2.55\left(\mathrm{~m}, 2 \mathrm{H}, \mathrm{OCHCH}_{2}\right), 2.00(\mathrm{t}, J=2.8 \mathrm{~Hz}, 1 \mathrm{H}, \mathrm{C} \equiv \mathrm{CH})$.

${ }^{13} \mathrm{C}$ NMR $\left(100 \mathrm{~Hz}, \mathrm{CDCl}_{3}\right): \delta=159.3,134.6,127.0,113.8,80.8,72.0$, $70.9,55.3,29.4$.

The spectra were in accordance with the previously reported data. ${ }^{21}$

\section{1-(3-Methoxyphenyl)but-3-yn-1-ol (3h)}

Yield: $165 \mathrm{mg}$ (93\%); yellow oil.

${ }^{1} \mathrm{H}$ NMR $\left(400 \mathrm{~Hz}, \mathrm{CDCl}_{3}\right): \delta=7.23-7.18\left(\mathrm{~m}, 1 \mathrm{H}_{\mathrm{arom}}\right), 6.90-6.88(\mathrm{~m}, 2$ $\left.\mathrm{H}_{\mathrm{arom}}\right), 6.79-6.76\left(\mathrm{~m}, 1 \mathrm{H}_{\mathrm{arom}}\right), 4.79\left(\mathrm{t}, J=6.4 \mathrm{~Hz}, 1 \mathrm{H}, \mathrm{OCHCH}_{2}\right), 3.75(\mathrm{~s}$, $\left.3 \mathrm{H}, \mathrm{OCH}_{3}\right), 2.58-2.56\left(\mathrm{~m}, 2 \mathrm{H}, \mathrm{OCHCH}_{2}\right), 2.01(\mathrm{t}, J=2.8 \mathrm{~Hz}, 1 \mathrm{H}, \mathrm{C} \equiv \mathrm{CH})$. ${ }^{13} \mathrm{C}$ NMR $\left(100 \mathrm{~Hz}, \mathrm{CDCl}_{3}\right): \delta=160.0,144.4,129.8,118.3,113.7,111.5$, $80.9,72.5,71.3,55.5,29.7$.

The spectra were in accordance with the previously reported data. ${ }^{21}$

\section{1-(2-Methoxyphenyl)but-3-yn-1-ol (3i)}

Yield: $167 \mathrm{mg}$ (94\%); colorless oil.

${ }^{1} \mathrm{H}$ NMR $\left(400 \mathrm{MHz}, \mathrm{CDCl}_{3}\right): \delta=7.34\left(\mathrm{dd}, J=7.6,1.6 \mathrm{~Hz}, 1 \mathrm{H}_{\mathrm{arom}}\right), 7.23-$ $7.18\left(\mathrm{~m}, 1 \mathrm{H}_{\text {arom }}\right), 6.91$ (dd, $\left.J=8.0,7.2 \mathrm{~Hz}, 1 \mathrm{H}_{\text {arom }}\right), 6.82(\mathrm{~d}, J=8.0 \mathrm{~Hz}, 1$ $\left.\mathrm{H}_{\text {arom }}\right), 5.01\left(\mathrm{dd}, J=7.6,4.8 \mathrm{~Hz}, 1 \mathrm{H}, \mathrm{OCHCH}_{2}\right), 3.79\left(\mathrm{~s}, 3 \mathrm{H}, \mathrm{OCH}_{3}\right), 2.70$ (ddd, $J=16.8,4.8,2,8 \mathrm{~Hz}, 1 \mathrm{H}, \mathrm{OCHCH}_{2}$ ), 2.57 (ddd, $J=16.8,7.6,2.8 \mathrm{~Hz}$, $\left.1 \mathrm{H}, \mathrm{OCHCH}_{2}\right), 1.98(\mathrm{t}, J=2.8 \mathrm{~Hz}, 1 \mathrm{H}, \mathrm{C} \equiv \mathrm{CH})$.

${ }^{13} \mathrm{C}$ NMR $\left(100 \mathrm{MHz}, \mathrm{CDCl}_{3}\right): \delta=156.5,130.6,129.1,127.1,121.0$, 110.7, 81.6, 70.7, 69.3, 55.6, 27.7.
The spectra were in accordance with the previously reported data. ${ }^{22}$

\section{1-Phenyl-3-butyn-1-ol (3j)}

Yield: $106 \mathrm{mg}$ (72\%); colorless oil.

${ }^{1} \mathrm{H}$ NMR $\left(400 \mathrm{MHz}, \mathrm{CDCl}_{3}\right): \delta=7.34-7.24\left(\mathrm{~m}, 5 \mathrm{H}_{\mathrm{arom}}\right), 4.82(\mathrm{t}, J=6.4$ $\left.\mathrm{Hz}, 1 \mathrm{H}, \mathrm{OCHCH}_{2}\right), 2.60-2.57\left(\mathrm{~m}, 2 \mathrm{H}, \mathrm{OCHCH}_{2}\right), 2.10$ (br s, $\left.1 \mathrm{H}, \mathrm{OH}\right)$, $2.01(\mathrm{t}, J=2.8 \mathrm{~Hz}, 1 \mathrm{H}, \mathrm{C} \equiv \mathrm{CH})$.

${ }^{13} \mathrm{C}$ NMR $\left(100 \mathrm{MHz}, \mathrm{CDCl}_{3}\right): \delta=142.5,128.4,127.9,125.7,80.7,72.0$, 70.7, 29.1.

The spectra were in accordance with the previously reported data. ${ }^{21}$

\section{1-(Naphth-2-yl)but-3-yn-1-ol (3k)}

Yield: $188 \mathrm{mg}$ (95\%); yellow oil.

${ }^{1} \mathrm{H}$ NMR $\left(400 \mathrm{MHz}, \mathrm{CDCl}_{3}\right): \delta=7.79-7.75\left(\mathrm{~m}, 4 \mathrm{H}_{\mathrm{arom}}\right), 7.44-7.40(\mathrm{~m}, 2$ $\left.\mathrm{H}_{\text {arom }}\right), 7.29\left(\mathrm{~s}, 1 \mathrm{H}_{\text {arom }}\right), 4.98\left(\mathrm{t}, J=6.0 \mathrm{~Hz}, 1 \mathrm{H}, \mathrm{OCHCH}_{2}\right), 2.68-2.66(\mathrm{~m}$, $2 \mathrm{H}, \mathrm{OCHCH}_{2}$ ), 2.41 (br s, $\left.1 \mathrm{H}, \mathrm{OH}\right), 2.01(\mathrm{t}, J=2.8 \mathrm{~Hz}, 1 \mathrm{H}, \mathrm{C} \equiv \mathrm{CH}$ ).

${ }^{13} \mathrm{C}$ NMR $\left(100 \mathrm{MHz}, \mathrm{CDCl}_{3}\right): \delta=139.8,133.13,133.09,128.3,128.0$, $127.7,126.2,126.0,124.6,123.7,80.6,72.4,71.1,29.4$

The spectra were in accordance with the previously reported data. ${ }^{21}$

\section{1-(2-Furyl)but-3-yn-1-ol (31)}

Yield: $101 \mathrm{mg}$ (73\%); yellow oil.

${ }^{1} \mathrm{H}$ NMR $\left(300 \mathrm{MHz}, \mathrm{CDCl}_{3}\right): \delta=7.32\left(\mathrm{t}, J=1.2 \mathrm{~Hz}, 1 \mathrm{H}_{\text {het }}\right), 6.28(\mathrm{~d}, J=1.2$ $\mathrm{Hz}, 2 \mathrm{H}_{\text {het }}$ ), $4.82\left(\mathrm{t}, J=6.0 \mathrm{~Hz}, 1 \mathrm{H}, \mathrm{OCHCH}_{2}\right), 2.71$ (dd, $J=6.0,2.4 \mathrm{~Hz}, 2$ $\left.\mathrm{H}, \mathrm{OCHCH}_{2}\right), 2.26(\mathrm{~s}, 1 \mathrm{H}, \mathrm{OH}), 2.01(\mathrm{t}, J=2.4 \mathrm{~Hz}, 1 \mathrm{H}, \mathrm{C} \equiv \mathrm{CH})$.

${ }^{13} \mathrm{C} \mathrm{NMR}\left(75 \mathrm{MHz}, \mathrm{CDCl}_{3}\right): \delta=154.6,142.3,110.2,106.6,79.8,71.1$, $66.1,26.1$

The spectra were in accordance with the previously reported data. ${ }^{21}$

\section{(E)-1-Phenylhex-1-en-5-yn-3-ol (3m)}

Yield: $156 \mathrm{mg}$ (90\%); yellow oil.

${ }^{1} \mathrm{H}$ NMR $\left(400 \mathrm{MHz}, \mathrm{CDCl}_{3}\right): \delta=7.33\left(\mathrm{~d}, J=7.6 \mathrm{~Hz}, 2 \mathrm{H}_{\mathrm{arom}}\right), 7.25(\mathrm{t}, J=$ $7.6 \mathrm{~Hz}, 1 \mathrm{H}_{\text {arom }}$ ), 7.20-7.16 (m, $\left.2 \mathrm{H}_{\text {arom }}\right), 6.60(\mathrm{~d}, J=16.0 \mathrm{~Hz}, 1 \mathrm{H}$, $\mathrm{CH}=\mathrm{CH}), 6.21$ (dd, $J=16.0,6.4 \mathrm{~Hz}, 1 \mathrm{H}, \mathrm{CH}=\mathrm{CH}), 4.43-4.39(\mathrm{~m}, 1 \mathrm{H}$, $\mathrm{OCHCH}_{2}$ ), 2.61 (ddd, $J=16.8,5.6,2.8 \mathrm{~Hz}, 1 \mathrm{H}, \mathrm{OCHCH}_{2}$ ), 2.55 (ddd, $J=$ $\left.16.8,6.0,2.4 \mathrm{~Hz}, 1 \mathrm{H}, \mathrm{OCHCH}_{2}\right), 2.02(\mathrm{t}, J=2.8 \mathrm{~Hz}, 1 \mathrm{H}, \mathrm{C} \equiv \mathrm{CH}), 1.89(\mathrm{br}$ $\mathrm{s}, 1 \mathrm{H}, \mathrm{OH})$.

${ }^{13} \mathrm{C}$ NMR $\left(100 \mathrm{MHz}, \mathrm{CDCl}_{3}\right): \delta=136.3,131.4,129.9,128.6,127.9$, 126.6, 80.2, 71.1, 70.7, 27.7.

The spectra were in accordance with the previously reported data. ${ }^{21}$

\section{Dec-1-yn-4-ol (3n)}

Yield: $125 \mathrm{mg}$ (80\%); colorless oil.

${ }^{1} \mathrm{H}$ NMR (300 MHz, $\left.\mathrm{CDCl}_{3}\right): \delta=3.80-3.72\left(\mathrm{~m}, 1 \mathrm{H}, \mathrm{OCHCH} \mathrm{CH}_{2}\right), 2.43$ (ddd, $\left.J=16.4,4.8,3.2 \mathrm{~Hz}, 1 \mathrm{H}, \mathrm{OCHCH}_{2} \mathrm{C} \equiv \mathrm{CH}\right), 2.33$ (ddd, $J=16.4,6.4,3.2 \mathrm{~Hz}$, $\left.1 \mathrm{H}, \mathrm{OCHCH}_{2} \mathrm{C} \equiv \mathrm{CH}\right), 2.06(\mathrm{t}, J=3.2 \mathrm{~Hz}, 1 \mathrm{H}, \mathrm{C} \equiv \mathrm{CH}), 1.97$ (br s, $\left.1 \mathrm{H}, \mathrm{OH}\right)$, 1.59-1,51 (m, $\left.2 \mathrm{H}, \mathrm{CH}_{2}\right), 1.37-1.24\left(\mathrm{~m}, 8 \mathrm{H}, 4 \times \mathrm{CH}_{2}\right), 0.89(\mathrm{t}, J=6.4 \mathrm{~Hz}$, $\left.3 \mathrm{H}, \mathrm{CH}_{3}\right)$.

${ }^{13} \mathrm{C}$ NMR $\left(75 \mathrm{MHz}, \mathrm{CDCl}_{3}\right): \delta=80.9,70.7,69.9,36.2,31.7,29.2,27.3$, 25.5, 22.6, 14.0.

The spectra were in accordance with the previously reported data. ${ }^{23}$

1-(5-Bromo-2-methoxyphenyl)but-3-yn-1-ol (3o)

Yield: $211 \mathrm{mg}$ (82\%); yellow oil. 
${ }^{1} \mathrm{H} \mathrm{NMR}\left(400 \mathrm{MHz}, \mathrm{CDCl}_{3}\right): \delta=7.48\left(\mathrm{~d}, J=2.4 \mathrm{~Hz}, 2 \mathrm{H}_{\mathrm{arom}}\right), 7.28(\mathrm{dd}, J=$ 8.4, $2.4 \mathrm{~Hz}, 1 \mathrm{H}_{\text {arom }}$ ), 6.67 (d, $J=8.8 \mathrm{~Hz}, 1 \mathrm{H}_{\text {arom }}$ ), 4.98 (dd, $J=7.6,4.8$ $\mathrm{Hz}, 1 \mathrm{H}, \mathrm{OCHCH}_{2}$ ), 3.75 (s, $3 \mathrm{H}, \mathrm{CH}_{3}$ ), 2.67 (ddd, $J=16.8,7.2,2.8 \mathrm{~Hz}, 1$ $\mathrm{H}, \mathrm{OCHCH}_{2}$ ), 2.48 (ddd, $\left.J=16.8,7.6,2.4 \mathrm{~Hz}, 1 \mathrm{H}, \mathrm{OCHCH}_{2}\right), 2.00(\mathrm{t}, J=$ $2.8 \mathrm{~Hz}, 1 \mathrm{H}, \mathrm{C} \equiv \mathrm{CH})$.

${ }^{13} \mathrm{C}$ NMR $\left(100 \mathrm{MHz}, \mathrm{CDCl}_{3}\right): \delta=155.1,132.5,131.25,129.6,113.2$, 112.0, 80.7, 70.9, 67.7, 55.5, 27.4 .

The spectra were in accordance with the previously reported data. ${ }^{24}$

\section{Methyl 4-(1-Hydroxybut-3-ynyl)benzoate (3p)}

Yield: $175 \mathrm{mg}$ (85\%); yellow oil.

${ }^{1} \mathrm{H}$ NMR (400 MHz, $\mathrm{CDCl}_{3}$ ): $\delta=7.95\left(\mathrm{~d}, J=8.8 \mathrm{~Hz}, 2 \mathrm{H}_{\mathrm{arom}}\right), 7.39(\mathrm{~d}, J=$ $\left.8.8 \mathrm{~Hz}, 2 \mathrm{H}_{\text {arom }}\right), 4.86$ (t, $\left.J=6.0 \mathrm{~Hz}, 1 \mathrm{H}_{\text {arom }}\right), 3.84\left(\mathrm{~s}, 3 \mathrm{H}, \mathrm{CH}_{3}\right), 2.61$ (ddd, $J=16.8,8.0,2.8 \mathrm{~Hz}, 1 \mathrm{H}, \mathrm{OCHCH}_{2}$ ), 2.55 (ddd, $J=16.8,7.2,2.8 \mathrm{~Hz}$, $\left.1 \mathrm{H}, \mathrm{OCHCH}_{2}\right), 2.01(\mathrm{t}, J=2.8 \mathrm{~Hz}, 1 \mathrm{H}, \mathrm{C} \equiv \mathrm{CH})$.

${ }^{13} \mathrm{C}$ NMR $\left(100 \mathrm{MHz}, \mathrm{CDCl}_{3}\right): \delta=166.8,147.4,129.8,129.7,125.7,80.0$, 71.8, 71.4, 52.1, 29.4 .

The spectra were in accordance with the previously reported data. ${ }^{6 a}$

\section{4-(1-Hydroxybut-3-ynyl)-2-methoxyphenol (3q)}

Yield: $136 \mathrm{mg}$ (70\%); yellow oil.

${ }^{1} \mathrm{H}$ NMR $\left(400 \mathrm{MHz}, \mathrm{CDCl}_{3}\right): \delta=7.96\left(\mathrm{~d}, J=2.0 \mathrm{~Hz}, 1 \mathrm{H}_{\mathrm{arom}}\right), 6.89(\mathrm{~d}, J=$ $\left.8.0 \mathrm{~Hz}, 1 \mathrm{H}_{\text {arom }}\right), 6.85$ (dd, $\left.J=8.4,1.6 \mathrm{~Hz}, 1 \mathrm{H}_{\text {arom }}\right), 4.81(\mathrm{t}, J=6.4 \mathrm{~Hz}, 1 \mathrm{H}$, $\left.\mathrm{OCHCH}_{2}\right), 3.90\left(\mathrm{~s}, 3 \mathrm{H}, \mathrm{CH}_{3}\right), 2.64-2.62\left(\mathrm{~m}, 2 \mathrm{H}, \mathrm{OCHCH}_{2}\right), 2.44$ (br s, 1 $\mathrm{H}, \mathrm{PhOH}), 2.08(\mathrm{t}, J=2.8 \mathrm{~Hz}, 1 \mathrm{H}, \mathrm{C} \equiv \mathrm{CH})$.

${ }^{13} \mathrm{C}$ NMR $\left(100 \mathrm{MHz}, \mathrm{CDCl}_{3}\right): \delta=146.5,145.3,134.5,118.8,114.1$, 108.23, 80.8, 72.2, 70.9, 55.9, 29.4 .

The spectra were in accordance with the previously reported data. ${ }^{25}$

\section{4-(1-Hydroxybut-3-yn-yl)benzonitrile (3r)}

Yield: $160 \mathrm{mg}$ (92\%); white solid; $\mathrm{mp} 120-122{ }^{\circ} \mathrm{C}$.

${ }^{1} \mathrm{H}$ NMR $\left(400 \mathrm{MHz}, \mathrm{CDCl}_{3}\right): \delta=7.58\left(\mathrm{~d}, J=8.0 \mathrm{~Hz}, 2 \mathrm{H}_{\mathrm{arom}}\right), 7.45(\mathrm{~d}, J=$ $\left.8.4 \mathrm{~Hz}, 2 \mathrm{H}_{\text {arom }}\right), 4.86\left(\mathrm{t}, J=6.0 \mathrm{~Hz}, 1 \mathrm{H}, \mathrm{OCHCH}_{2}\right), 2.63-2.51(\mathrm{~m}, 2 \mathrm{H}$, $\left.\mathrm{OCHCH}_{2}\right), 2.11(\mathrm{~d}, J=2.4 \mathrm{~Hz}, 1 \mathrm{H}, \mathrm{C} \equiv \mathrm{CH})$.

${ }^{13} \mathrm{C}$ NMR $\left(100 \mathrm{MHz}, \mathrm{CDCl}_{3}\right): \delta=147.5,132.2,126.5,118.6,111.6,79.5$, $71.8,71.4,29.4$.

The spectra were in accordance with the previously reported data. ${ }^{6 a}$

\section{1-[4-(1-Hydroxybut-3-yn-1-yl)phenyl]ethanone (3s)}

Yield: $179 \mathrm{mg}$ (94\%); yellow oil.

${ }^{1} \mathrm{H}$ NMR $\left(300 \mathrm{MHz}, \mathrm{CDCl}_{3}\right): \delta=7.88\left(\mathrm{~d}, J=11.2 \mathrm{~Hz}, 2 \mathrm{H}_{\mathrm{arom}}\right), 7.43(\mathrm{~d}, J=$ $11.2 \mathrm{~Hz}, 2 \mathrm{H}_{\text {arom }}$ ), 4.87 (dd, $\left.J=8.8,8.4 \mathrm{~Hz}, 1 \mathrm{H}, \mathrm{OCHCH}_{2}\right), 2.61-2.57(\mathrm{~m}$, $\left.2 \mathrm{H}, \mathrm{OCHCH}_{2}\right), 2.53\left(\mathrm{~s}, 3 \mathrm{H}, \mathrm{CH}_{3}\right), 2.47(\mathrm{br} \mathrm{s}, 1 \mathrm{H}, \mathrm{OH}), 2.09(\mathrm{t}, J=3.0 \mathrm{~Hz}$, $1 \mathrm{H}, \mathrm{C} \equiv \mathrm{CH}$ ).

${ }^{13} \mathrm{C}$ NMR $\left(75 \mathrm{MHz}, \mathrm{CDCl}_{3}\right): \delta=197.8,147.6,136.7,128.6,125.9,79.9$, 71.6, 71.5, 29.4, 26.7.

HRMS (ESI, MeOH- $\mathrm{H}_{2} \mathrm{O}$ ): $\mathrm{m} / z$ calcd for $\mathrm{C}_{12} \mathrm{H}_{12} \mathrm{O}_{2}[\mathrm{M}-\mathrm{H}]^{+}:$187.0765; found: 187.0748 .

\section{Acknowledgment}

The authors gratefully acknowledge CNPq (478947/2013-5 and 482299/2013-4), FACEPE (PRONEX APQ-0859-1.06/08), CAPES, and inct-INAMI for financial support. The authors are also thankful to CNPq for their fellowships.

\section{Supporting Information}

Supporting information for this article is available online at http://dx.doi.org/10.1055/s-0034-1379163. Included are additional experimental procedures and ${ }^{1} \mathrm{H},{ }^{13} \mathrm{C},{ }^{19} \mathrm{~F}$, and ${ }^{11} \mathrm{~B}$ NMR for all synthesized compounds.

\section{References}

(1) Yus, M.; Gonzalez-Gomez, J. C.; Foubelo, F. Chem. Rev. 2011, 111, 7774.

(2) Ding, C-H.; Hou, X-L. Chem. Rev. 2011, 111, 1914.

(3) (a) Wisniewska, H. M.; Jarvo, E. R. J. Org. Chem. 2013, 78, 11629. (b) Bejjani, J.; Botuha, C.; Chemla, F.; Ferreira, F.; Magnus, S.; Perez-Luna, A. Organometallics 2012, 31, 4876.

(4) (a) Fandrick, D. R.; Saha, J.; Fandrick, K. R.; Sanyal, S.; Ogikubo, J.; Lee, H.; Roschangar, F.; Song, J. H. J.; Senanayake, C. H. Org. Lett. 2011, 13, 5616. (b) Chemla, F.; Ferreira, F. Synlett 2006, 2613. (c) Chemla, F.; Ferreira, F.; Hebbe, V.; Stercklen, E. Eur. J. Org. Chem. 2002, 1385. (d) Poisson, J-F.; Normant, J. F. J. Org. Chem. 2000, 65, 6553. (e) Zweifel, G.; Hahn, G. J. Org. Chem. 1984, 49, 4565.

(5) (a) Millan, A.; de Cienfuegos, L. A.; Martin-Lasanta, A.; Campana, A. G.; Cuerva, J. M. Adv. Synth. Catal. 2011, 353, 73. (b) Furata, K.; Ishiguro, M.; Haruta, R.; Ikeda, N.; Yamamoto, H. Bull. Chem. Soc. Jpn. 1984, 57, 2768. (c) Ishiguro, M.; Ikeda, N.; Yamamoto, H. J. Org. Chem. 1982, 47, 2225.

(6) (a) Guo, L. N.; Gao, H. J.; Mayer, P.; Knochel, P. Chem. Eur. J. 2010, 16, 9829. (b) Pearson, N. R.; Hahn, G.; Zweifel, G. J. Org. Chem. 1982, 47, 3364. (c) Guillerm-dron, D.; Capmau, M. L.; Chodkiewicz, W. Tetrahedron Lett. 1972, 37.

(7) (a) Reich, H. J.; Holladay, J. E.; Walker, T. G.; Thompson, J. L. J. Am. Chem. Soc. 1999, 121, 9769. (b) Bour, C.; Suffert, J. Eur. J. Org. Chem. 2006, 1390. (c) Corey, E. J.; Rucker, C. Tetrahedron Lett. 1982, 23, 719.

(8) (a) Lambert, C.; von Ragué Schleyer, P. Angew. Chem., Int. Ed. Engl. 1994, 33, 1129. (b) Saniere-Karila, M.; Capmau, M. L.; Chodkiewicz, W. Bull. Soc. Chim. Fr. 1973, 3371.

(9) (a) Brawn, R. A.; Panek, J. S. Org. Lett. 2007, 9, 2689. (b) Sammtleben, F.; Noltemeyer, M.; Bruckner, R. Tetrahedron Lett. 1997, 38, 3893. (c) Danheiser, R. L.; Carini, D. J.J. Org. Chem. 1980, 45, 3925.

(10) (a) Chatterjee, P. N.; Roy, S. J. Org. Chem. 2010, 75, 4413. (b) Banerjee, M.; Roy, S. Org. Lett. 2004, 6, 2137. (c) McCluskey, A.; Muderawan, M.; Young, D. J. Synlett 1998, 909. (d) Mukaiyama, T.; Harada, T. Chem. Lett. 1981, 621. (e) Lequan, M.; Guillerm, G. J. Organomet. Chem. 1973, 54, 153.

(11) (a) Bhakta, U.; Sullivan, E.; Hall, D. G. Tetrahedron 2014, 70, 678. (b) Nowrouzi, F.; Batey, R. A. Angew. Chem. Int. Ed. 2013, 52, 892. (c) Xing, C-H.; Liao, Y-X.; Zhang, Y.; Sabarova, D.; Bassous, M.; Hu, Q-S. Eur. J. Org. Chem. 2012, 1115. (d) Hirayama, L. C.; Haddad, T. D.; Oliver, A. G.; Singaram, B. J. Org. Chem. 2012, 77, 4342. (e) Jain, P.; Wang, H.; Houk, K. N.; Antilla, J. C. Angew. Chem. Int. Ed. 2012, 51, 1391. (f) Chen, M.; Roush, W. R. J. Am. Chem. Soc. 2012, 134, 10947. (g) Reddy, L. R. Org. Lett. 2012, 14, 1142. (h) Brown, H. C.; Khire, U. R.; Narla, G. J. J. Org. Chem. 1995, 60, 8130. (i) Farve, E.; Gaudemar, M. J. Organomet. Chem. 1974, 76, 297.

(12) Tri-n-butyltin Compounds [MAK Value Documentation, 1990]. The MAK Collection for Occupational Health and Safety; WileyVCH: Weinheim, 2012, 316.

(13) Molander, G. A.; Figueroa, R. Aldrichimica Acta 2005, 38, 49. 
Synthesis

(14) Science of Synthesis: Boron Compounds; Vol. 6; Kaufmann, D. E.; Matteson, D. S., Eds.; Thieme: Stuttgart, 2005.

(15) (a) Bernardini, R.; Oliva, A.; Paganelli, A.; Menta, E. Chem. Lett. 2009, 38, 750. (b) Matteson, D. S. Tetrahedron 1998, 54, 10555.

(16) Oliveira, R. A.; Silva, R. O.; Molander, G. A.; Menezes, P. H. Magn. Res. Chem. 2009, 47, 873.

(17) Petrillo, D. E.; Kohli, R. K.; Molander, G. A. J. Am. Soc. Mass Spectrom. 2007, 18, 404.

(18) Trost, B. M. Science 1991, 254, 1471.

(19) Oliveira, R. A.; Savegnago, L.; Jesse, C. R.; Menezes, P. H.; Molander, G. A.; Nogueira, C. W. Basic Clin. Pharmacol. Toxicol. 2009, 104, 448.
(20) Couto, T. R.; Freitas, J. C. R.; Cavalcanti, I. H.; Oliveira, R. A.; Menezes, P. H. Tetrahedron 2013, 69, 7006.

(21) Li, Q. R.; Gu, C. Z.; Yin, H. Chin. J. Chem. 2006, 24, 72.

(22) Chen, J.; Captain, B.; Takenaka, N. Org. Lett. 2011, 13, 1654.

(23) Ma, X.; Wang, J. X.; Li, S.; Wang, K. H.; Huang, D. Tetrahedron 2009, 65, 8683.

(24) Commercial product, available from Aurora Fine Chemicals, USA; CAS Number: 1342503-79-0.

(25) Wu, S.; Huang, B.; Gao, X. Synth. Commun. 1990, 20, 1279.

This article differs from the e-first online version only in its layout; no content has been changed. 